\title{
The molecular basis of mouse sperm-zona pellucida binding: a still unresolved issue in developmental biology
}

\author{
Gary F Clark \\ Drive, Columbia, Missouri 65211, USA \\ Correspondence should be addressed to G F Clark; Email: clarkgf@health.missouri.edu
}

Department of Obstetrics, Gynecology and Women's Health, University of Missouri School of Medicine, 1 Hospital

\begin{abstract}
During murine fertilization, sperm bind to the specialized extracellular matrix of the egg, known as the zona pellucida (ZP). This matrix is composed of three major glycoproteins designated ZP1, ZP2, and ZP3. Three models for sperm-ZP binding are now under consideration. The domain-specific model posits that adhesion relies primarily on interactions between $\mathbf{N}$-glycans located within the $\mathrm{C}$-terminal domain of ZP3 and a lectin-like egg-binding protein in the sperm plasma membrane. However, this model does not explain recent results obtained in studies with $Z P 2^{m u t}$ mice. In the supramolecular structure model, sperm bind to a three-dimensional zona matrix that depends on the cleavage status of ZP2. This paradigm does not explain the potent inhibitory effect of specific carbohydrate sequences or a C-terminal glycopeptide (gp55) derived from ZP3. Recently, O-glycans linked at Thr ${ }^{155}$ and $\mathrm{Thr}^{162}$ of ZP3 were implicated as potential ligands that mediate initial sperm-ZP binding. This novel model will be reviewed. A major challenge is to develop an alternate model for sperm-ZP binding that fits as much of the data as possible. Such a model is presented in this review. This paradigm could explain how the inability to cleave $Z P 2^{m u t}$ in $Z P 2^{\text {mut }}$ mice could result in continued sperm binding to two-cell stage embryos without the formation of a supramolecular binding complex. These novel insights should guide future experiments that will eventually determine the molecular basis underlying gamete binding in the mouse and other eutherian mammals.
\end{abstract}

Reproduction (2011) 142 377-381

\section{Introduction}

Like all mammalian eggs, the mouse oocyte is surrounded by a specialized extracellular matrix known as the zona pellucida (ZP). The first committed step in mouse reproduction is the binding of sperm to this matrix. The $Z P$ is composed of three major glycoproteins designated ZP1, ZP2, and ZP3 (Bleil \& Wassarman 1980b). Early studies with these purified glycoproteins led to the development of a classical model for sperm-ZP binding. ZP3 is the only glycoprotein purified from this matrix that potently inhibits murine sperm-ZP binding and induces the acrosome reaction in free swimming sperm in vitro (Bleil \& Wassarman 1980a, 1983). Based on these observations, sperm were proposed to bind to ZP3, and rapidly undergo the acrosome reaction (Bleil \& Wassarman 1983). During this reaction, the plasma membrane fuses with the outer acrosomal membrane, forming membrane blebs that detach from the head region of sperm (Austin 1977). This activation process exposes the inner acrosomal membrane. In this classical model, receptors on this membrane bind to ZP2 on the zona to mediate secondary binding (Bleil et al. 1988). Sperm penetrate the $\mathrm{ZP}$, enter the perivitelline space, and fuse with the oocyte, completing the binding steps required for fertilization. Many different adhesion molecules that mediate initial sperm-ZP binding have been proposed since 1980 (Tulsiani et al. 1997, Clark \& Dell 2006, Nixon et al. 2009).

\section{A novel $\boldsymbol{O}$-glycosylation domain that mediates murine gamete binding}

Early studies implicated carbohydrate recognition in sperm binding. The digestion of ZP3 with pronase released small glycopeptides that inhibited sperm-ZP binding nearly as effectively as the intact glycoprotein (Florman et al. 1984). ZP3-derived $O$-glycans ranging in size between 3.4 and $4.5 \mathrm{kDa}$ also inhibited sperm-ZP binding (Florman \& Wassarman 1985). Subsequent mutagenesis of presumed O-glycosylation sites at $\mathrm{Ser}^{332}$ and $\mathrm{Ser}^{334}$ of ZP3 resulted in the loss of its sperm receptor activity, indicating the presence of an $O$-glycosylation domain that mediated binding (Chen et al. 1998). This model for O-glycan participation in mouse sperm-ZP binding was widely accepted, and appeared in a major cell biology textbook (Alberts et al. 2002). 
Careful analysis of the results obtained in previous studies now enables new conclusions to be drawn about the role of $O$-glycans in sperm-ZP binding. Ultrasensitive MS studies indicated that the mass of ZP3-derived $O$-glycans does not exceed $1.5 \mathrm{kDa}$, far less than the mass of the putative $O$-glycans that reportedly inhibited sperm-ZP binding (3.4-4.5 kDa; Florman \& Wassarman 1985, Dell et al. 2003). Modern glycoproteomic analyses have also confirmed that ZP3 is not $O$-glycosylated at either $\mathrm{Ser}^{332}$ or Ser ${ }^{334}$ (Fig. 1; Boja et al. 2003, Chalabi et al. 2006). These results are incompatible with the hypothesis that an $O$-glycosylated region at these positions mediates sperm binding (Chen et al. 1998).

The digestion of ZP3 with papain at a single site released $\sim 26$ and $55 \mathrm{kDa}$ products from the $\mathrm{N}$ - and C-terminal regions of this glycoprotein respectively (Fig. 1; Rosiere \& Wassarman 1992). The $\sim 55 \mathrm{kDa}$ C-terminal product (gp55) but not the $\sim 26 \mathrm{kDa}$ product inhibited sperm-ZP binding and induced the acrosome reaction nearly as effectively as intact ZP3 (Rosiere \& Wassarman 1992, Litscher \& Wassarman 1996). Modern glycoproteomic analyses have confirmed that gp55 is not O-glycosylated (Fig. 1; Boja et al. 2003, Chalabi et al. 2006). N-glycanase digestion of gp55 generated a $21 \mathrm{kDa}$ product that retains the same sperm receptor and acrosome-inducing activities as the intact glycopeptide (Fig. 1; Litscher \& Wassarman 1996). Modern
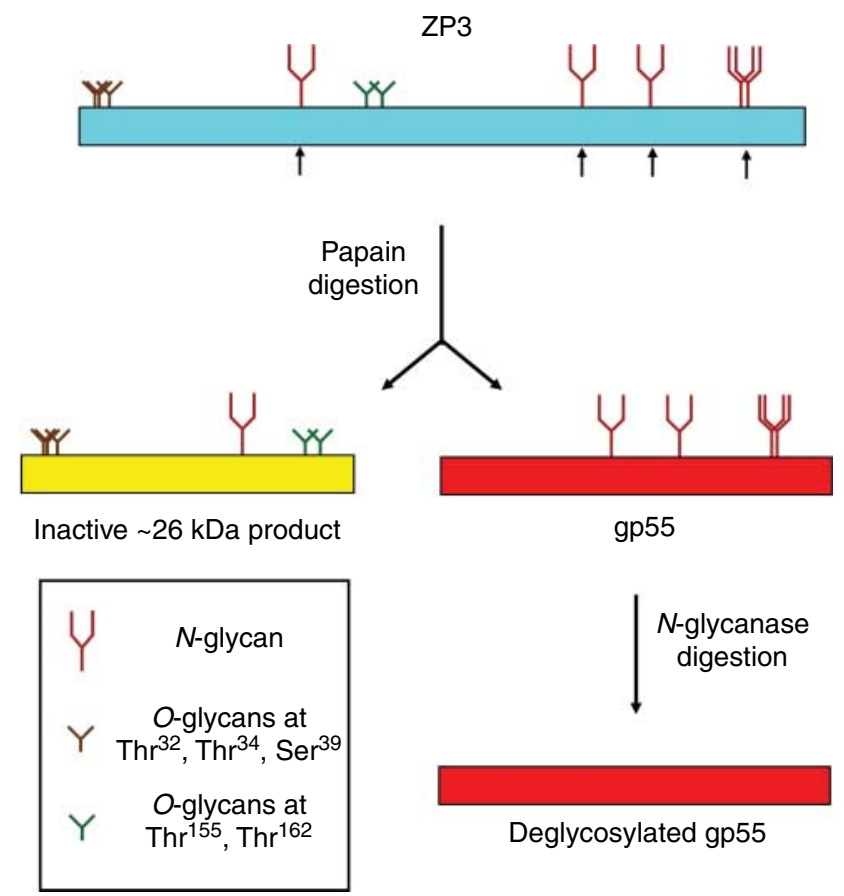

Figure 1 Glycoproteomic map of ZP3 and digestion products discussed in the text. The positions of the $\mathrm{N}$ - and $\mathrm{O}$-glycosylation sites are based on results obtained in two previous studies (Boja et al. 2003, Chalabi et al. 2006). All of the $O$-glycans are located on the $26 \mathrm{kDa} \mathrm{N}$-terminal product obtained by papain digestion, whereas only $\mathrm{N}$-glycans are associated with gp55. The small arrows point to $\mathrm{N}$-glycosylation sites located at $\mathrm{Asn}^{146}, \mathrm{Asn}^{273}, \mathrm{Asn}^{304}$, and $\mathrm{Asn}^{327} / \mathrm{Asn}^{330}$. glycoproteomic analyses confirm that this $21 \mathrm{kDa}$ product does not express any $\mathrm{N}$ - or $\mathrm{O}$-glycans (Fig. 1 ; Boja et al. 2003, Chalabi et al. 2006). The inhibitory effect of this deglycosylated product derived from the C-terminal end of ZP3 is major evidence that supports the domain-specific hypothesis for sperm binding (Clark 2010, 2011).

Recently, O-glycans positioned at $\mathrm{Thr}^{155}$ and $\mathrm{Thr}^{162}$ were proposed to constitute an alternate $O$-glycosylation region in ZP3 that mediates sperm-ZP binding (Visconti $\&$ Florman 2010). However, these sites are present in the $\sim 26 \mathrm{kDa}$ glycopeptide obtained after the digestion of ZP3 with papain (Fig. 1). This product completely lacked sperm receptor activity, unlike gp55 (Rosiere \& Wassarman 1992). The mismatch in the observed mass of the inhibitory O-glycans in ZP3 (3.4-4.5 kDa) compared with the actual mass of the $O$-glycans derived from this glycoprotein $(<1.5 \mathrm{KDa})$ also does not support this model for sperm binding (Florman \& Wassarman 1985, Dell et al. 2003). In summary, the existing evidence does not support any role for $O$-glycans in mediating sperm-ZP binding.

\section{An alternate model for mouse sperm-ZP binding that fits the data}

The supramolecular complex and domain-specific models for murine gamete binding have been compared in depth in previous reviews (Dean 2004, Clark 2010, 2011). The supramolecular complex model does not explain the inhibitory effect of oligosaccharides and glycoconjugates on sperm-ZP binding (Litscher et al. 1995, Clark 2011). It also does not provide a basis for the potent inhibitory effect of gp55 or its $21 \mathrm{kDa}$ deglycosylated analog on this interaction (Litscher \& Wassarman 1996). The domain-specific model addresses these specific deficiencies because of its major reliance on carbohydrate-mediated interactions. However, this model does not explain how sperm readily bind to two-cell stage embryos from $Z P 2^{\text {mut }}$ mice, but not to similar embryos from wild-type mice (Gahlay et al. 2010).

Recent studies have also called into question the classical model for mouse sperm-egg binding and induction of the acrosome reaction discussed previously. Dean (2004) have shown that mouse sperm do not rapidly undergo the acrosome reaction after binding to the ZP (Gahlay et al. 2010). On the other hand, only acrosome-reacted sperm were observed in the perivitelline space. Hirohashi and coworkers recently reported that most sperm readily undergo the acrosome reaction before they encounter the ZP under in vitro conditions, during their interactions with cumulus cells (Jin et al. 2011, Yanagimachi 2011). These investigators also presented evidence indicating that acrosome-reacted sperm readily bind to the ZP (Jin et al. 2011). 
These results indicate that redundant mechanisms for sperm binding and the induction of the acrosome reaction could exist. Normally, sperm interact with cumulus cells, triggering the acrosome reaction, and the resulting acrosome-reacted sperm bind to the ZP (Jin et al. 2011). However, some sperm may not undergo the acrosome reaction during their penetration of the cumulus oophorus. Alternatively, some eggs could lose their cumulus cells before encountering sperm. The existence of a redundant system for binding and induction of the acrosome reaction mediated by the ZP would rescue such eggs, enabling their fertilization.

A potential cause for concern for this model is that the sperm that undergo the acrosome reaction after contact with the cumulus cells are labeled with enhanced green fluorescent protein (EGFP) in their acrosomes and a red fluorescent protein (DSRed2) in their mitochondria (Jin et al. 2011). Genetic manipulations of sperm have often led to unpredictable morphological and physiological changes (Baba et al. 1994, Lu \& Shur 1997, Nishimura et al. 2001, Ensslin et al. 2007, Lin et al. 2007). Because of these previous problems, some skepticism is warranted about the findings with these in vivo fluorescently tagged sperm (Jin et al. 2011). Certainly there is also compelling evidence that acrosome intact sperm penetrate the cumulus oophorus and bind to the ZP (Florman \& Storey 1982, Storey et al. 1984).

There is no question that purified ZP3 readily induces the acrosome reaction in sperm in vitro (Bleil \& Wassarman 1983). If the assumption is made that the genetic insertion of EGFP in mouse sperm does not affect their physiological responses, then ZP3 in the intact ZP likely induces the acrosome reaction in bound sperm, but at a far slower rate than is observed when sperm are exposed to purified ZP3 in vitro. A rational explanation for this difference is that the acrosome-inducing activity of ZP3 is somehow regulated in the zona matrix. Such regulation could be physiologically relevant, because slowing down the induction of the acrosome reaction could reduce the chance that polyspermy will occur.

A major challenge is to construct a paradigm that reconciles as much of the existing data obtained in mouse fertilization studies as possible. A scheme consistent with this goal is presented in Fig. 2. In this model, multiple adhesion proteins on sperm bind to both $\mathrm{N}$-glycans and accessible protein regions located within the C-terminal domain of ZP3 (Fig. 2A). These adhesion proteins are very likely localized to the detergent resistant membrane fraction in mouse sperm (Nixon et al. 2009). The ZP is composed of a polymer of ZP2-ZP3 dimers that form fibers that are cross-linked by ZP1 (Greve \& Wassarman 1985). In this new model, the close association of ZP2 and ZP3 limits the ability of ZP3 to induce the acrosome reaction in bound sperm (Fig. 2A). Acrosome-reacted sperm bind to ZP2, enabling these gametes to penetrate the zona, and fuse with the oocyte (Bleil et al. 1988). This fusion event triggers the release of a ZP2-specific protease and other enzymes from the cortical granules (Moller \& Wassarman 1989). ZP2 is cleaved by this protease, generating ZP2C (Fig. 2B). ZP2C dissociates from ZP3, making ZP3 accessible to a currently unknown modification that converts ZP3 to ZP3f. This modified form of ZP3 present in two-cell stage embryos reportedly does not support sperm binding or induce the acrosome reaction in vitro (Fig. 2B; Bleil \& Wassarman 1980a). Neither ZP3f nor ZP2C can mediate the binding of intact or acrosomereacted sperm to the ZP. The net result is that sperm do not bind to two-cell stage embryos from wild-type mice (Fig. 2B). However, in $Z P 2^{\text {mut }}$ mice, $Z P 2^{\text {mut }}$ remains uncleaved, and ZP3 remains closely associated with $Z P 2^{\text {mut }}$. This association prevents the conversion of ZP3 to $Z \mathrm{P} 3 \mathrm{f}$, and acrosome intact sperm readily bind to twocell stage embryos from $Z P 2^{\text {mut }}$ mice, as reported recently (Gahlay et al. 2010). This model for the modification of these ZP glycoproteins is operational whether acrosome intact or acrosome-reacted sperm are initially bound to the ZP.

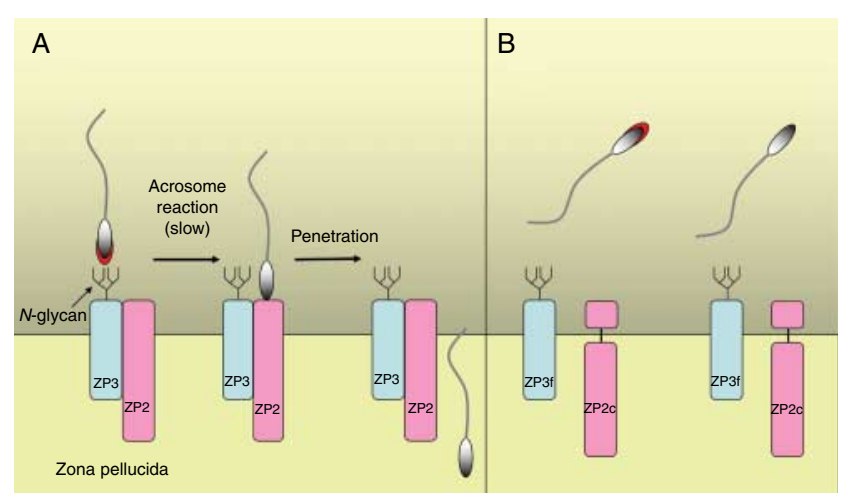

Figure 2 An alternate scheme for the sperm-ZP binding events leading to the fertilization and post-fertilization inhibition of sperm binding. Acrosome intact sperm have a red crescent on their head; acrosomereacted sperm do not. (A) In freshly ovulated eggs, ZP3 is in very close physical association with ZP2. This association likely inhibits the acrosome-inducing activity of ZP3. Sperm bind to N-glycans and other accessible sites for protein-protein interactions on ZP3, and slowly undergo the acrosome reaction. Acrosome-reacted sperm bind to ZP2 via their exposed inner acrosomal membranes, penetrate the zona, fuse with the oocyte, and fertilize the egg. (B) Immediately after fertilization, cortical granules release a ZP2-specific protease and other enzymes into the perivitelline space. This cortical granule protease clips ZP2 and converts it into the cleaved form (ZP2c) that no longer supports the binding of acrosome-reacted sperm. ZP3 dissociates from ZP2C, and undergoes a subtle modification that converts it into a form (ZP3f) that lacks sperm receptor and acrosome-inducing activity. Neither acrosome intact nor acrosome-reacted sperm can bind to ZP3f or ZP2C, consistent with the observation that two-cell stage embryos from wild-type mice do not bind sperm (Bleil \& Wassarman 1980a). However, in $Z P 2^{\text {mut }}$ mice, $Z P 2^{\text {mut }}$ remains uncleaved, and in close association with ZP3 after fertilization. ZP3 is not converted to ZP3f and two-cell stage embryos from $Z P 2^{\text {mut }}$ mice also bind sperm (Gahlay et al. 2010). 
The first step in the validation of this novel model will be to define the molecular differences that exist between ZP3 and ZP3f in unfertilized eggs and two-cell stage embryos respectively (Bleil \& Wassarman 1980a). The next step will be to demonstrate that the conversion of ZP3 to ZP3f does not occur in two-cell stage embryos derived from $Z P 2^{m u t}$ mice. Ultrasensitive mass spectrometric analyses will be essential to define these differences.

\section{Summary}

Currently, there are no substantive findings that support a role for $\mathrm{O}$-glycan recognition in mediating sperm-ZP binding. Neither the supramolecular complex model nor the domain-specific model for murine gamete binding can explain certain observations that have been made during the early events of fertilization. The novel scheme for sperm binding, induction of the acrosome reaction and subsequent post-fertilization events outlined in Fig. 2 is consistent with nearly all the existing data, including recent results obtained with $Z P 2^{\text {mut }}$ mice (Gahlay et al. 2010). More experiments will be required to validate a recent study performed with sperm that have been labeled with fluorescent proteins (Gahlay et al. 2010, Jin et al. 2011). Continued testing of these paradigms for sperm-ZP binding should eventually lead to a more thorough understanding of the molecular basis of this interaction in mice and other eutherian mammals.

\section{Declaration of interest}

The authors declare that there is no conflict of interest that could be perceived as prejudicing the impartiality of the review reported.

\section{Funding}

Studies related to mammalian sperm-egg interactions carried out by the author have been supported by the Life Sciences Mission Enhancement Reproductive Biology Program funded by the State of Missouri, the Jeffress Memorial Trust of Virginia, and the Breeden-Adams Foundation.

\section{References}

Alberts B, Johnson A, Lewis J, Raff M, Roberts K \& Walter P 2002 Molecular Biology of the Cell, 4 edn, pp 1151-1152. New York: Garland Science. Austin CR 1977 Spermatozoa and ova: the role of membranes in the fertilization process. In Mammalian Cell Membranes, 3rd edn, pp 206-230. Eds GA Jamieson\& DM Robinson. London: Butterworth.

Baba T, Azuma S, Kashiwabara S \& Toyoda Y 1994 Sperm from mice carrying a targeted mutation of the acrosin gene can penetrate the oocyte zona pellucida and effect fertilization. Journal of Biological Chemistry 269 31845-31849.
Bleil JD \& Wassarman PM 1980a Mammalian sperm-egg interaction: identification of a glycoprotein in mouse egg zonae pellucidae possessing receptor activity for sperm. Cell 20 873-882. (doi:10.1016/ 0092-8674(80)90334-7)

Bleil JD \& Wassarman PM $1980 b$ Structure and function of the zona pellucida: identification and characterization of the proteins of the mouse oocyte's zona pellucida. Developmental Biology 76 185-202. (doi:10.1016/0012-1606(80)90371-1)

Bleil JD \& Wassarman PM 1983 Sperm-egg interactions in the mouse: sequence of events and induction of the acrosome reaction by a zona pellucida glycoprotein. Developmental Biology 95 317-324. (doi:10. 1016/0012-1606(83)90032-5)

Bleil JD, Greve JM \& Wassarman PM 1988 Identification of a secondary sperm receptor in the mouse egg zona pellucida: role in maintenance of binding of acrosome-reacted sperm to eggs. Developmental Biology 128 376-385. (doi:10.1016/0012-1606(88)90299-0)

Boja ES, Hoodbhoy T, Fales HM \& Dean J 2003 Structural characterization of native mouse zona pellucida proteins using mass spectrometry. Journal of Biological Chemistry 278 34189-34202. (doi:10.1074/jbc. M304026200)

Chalabi S, Panico M, Sutton-Smith M, Haslam SM, Patankar MS, Lattanzio FA, Morris HR, Clark GF \& Dell A 2006 Differential $O$-glycosylation of a conserved domain expressed in murine and human ZP3. Biochemistry 45 637-647. (doi:10.1021/bi0512804)

Chen J, Litscher ES \& Wassarman PM 1998 Inactivation of the mouse sperm receptor, mZP3, by site-directed mutagenesis of individual serine residues located at the combining site for sperm. PNAS 95 6193-6197. (doi:10.1073/pnas.95.11.6193)

Clark GF 2010 The mammalian zona pellucida: a matrix that mediates both gamete binding and immune recognition. Systems Biology in Reproductive Medicine 56 349-364. (doi:10.3109/19396360903524812)

Clark GF 2011 Molecular models for sperm-oocyte binding. Glycobiology 21 3-5. (doi:10.1093/glycob/cwq159)

Clark GF \& Dell A 2006 Molecular models for murine sperm-egg binding. Journal of Biological Chemistry 281 13853-13856. (doi:10.1074/jbc. R600001200)

Dean J 2004 Reassessing the molecular biology of sperm-egg recognition with mouse genetics. Bioessays 26 29-38. (doi:10.1002/bies.10412)

Dell A, Chalabi S, Easton RL, Haslam SM, Sutton-Smith M, Patankar MS, Lattanzio F, Panico M, Morris HR \& Clark GF 2003 Murine and human ZP3 derived from mouse eggs express identical O-glycans. PNAS 100 15631-15636. (doi:10.1073/pnas.2635507100)

Ensslin MA, Lyng R, Raymond A, Copland S \& Shur BD 2007 Novel gamete receptors that facilitate sperm adhesion to the egg coat. Society of Reproduction and Fertility Supplement 63 367-383.

Florman HM \& Storey BT 1982 Mouse gamete interactions: the zona pellucida is the site of the acrosome reaction leading to fertilization in vitro. Developmental Biology 91 121-130. (doi:10.1016/0012-1606 (82) $90015-X)$

Florman HM \& Wassarman PM 1985 O-linked oligosaccharides of mouse egg ZP3 account for its sperm receptor activity. Cell 41 313-324. (doi:10. 1016/0092-8674(85)90084-4)

Florman HM, Bechtol KB \& Wassarman PM 1984 Enzymatic dissection of the functions of the mouse egg's receptor for sperm. Developmental Biology 106 243-255. (doi:10.1016/0012-1606(84)90079-4)

Gahlay G, Gauthier L, Baibakov B, Epifano O \& Dean J 2010 Gamete recognition in mice depends on the cleavage status of an egg's zona pellucida protein. Science 329 216-219. (doi:10.1126/science. 1188178)

Greve JM \& Wassarman PM 1985 Mouse egg extracellular coat is a matrix of interconnected filaments possessing a structural repeat. Journal of Molecular Biology 181 253-264. (doi:10.1016/0022-2836 (85)90089-0)

Jin M, Fujiwara E, Kakiuchi Y, Okabe M, Satouh Y, Baba SA, Chiba K \& Hirohashi N 2011 Most fertilizing mouse spermatozoa begin their acrosome reaction before contact with the zona pellucida during in vitro fertilization. PNAS 108 4892-4896. (doi:10.1073/pnas.1018202108)

Lin YN, Roy A, Yan W, Burns KH \& Matzuk MM 2007 Loss of zona pellucida binding proteins in the acrosomal matrix disrupts acrosome biogenesis and sperm morphogenesis. Molecular and Cellular Biology 27 6794-6805. (doi:10.1128/MCB.01029-07) 
Litscher ES \& Wassarman PM 1996 Characterization of mouse ZP3-derived glycopeptide, gp55, that exhibits sperm receptor and acrosome reactioninducing activity in vitro. Biochemistry 35 3980-3985. (doi:10.1021/ bi952722m)

Litscher ES, Juntunen K, Seppo A, Penttila L, Niemela R, Renkonen O \& Wassarman PM 1995 Oligosaccharide constructs with defined structures that inhibit binding of mouse sperm to unfertilized eggs in vitro. Biochemistry 34 4662-4669. (doi:10.1021/bi00014a020)

Lu Q \& Shur BD 1997 Sperm from $\beta 1$,4-galactosyltransferase-null mice are refractory to ZP3-induced acrosome reactions and penetrate the zona pellucida poorly. Development 124 4121-4131.

Moller CC \& Wassarman PM 1989 Characterization of a proteinase that cleaves zona pellucida glycoprotein ZP2 following activation of mouse eggs. Developmental Biology 132 103-112. (doi:10.1016/00121606(89)90209-1)

Nishimura H, Cho C, Branciforte DR, Myles DG \& Primakoff P 2001 Analysis of loss of adhesive function in sperm lacking cyritestin or fertilin- $\beta$. Developmental Biology 233 204-213. (doi:10.1006/dbio. 2001.0166)

Nixon B, Bielanowicz A, McLaughlin EA, Tanphaichitr N, Ensslin MA \& Aitken RJ 2009 Composition and significance of detergent resistant membranes in mouse spermatozoa. Journal of Cellular Physiology 218 122-134. (doi:10.1002/jcp.21575)
Rosiere TK \& Wassarman PM 1992 Identification of a region of mouse zona pellucida glycoprotein mZP3 that possesses sperm receptor activity. Developmental Biology 154 309-317. (doi:10.1016/0012-1606(92) 90070-W)

Storey BT, Lee MA, Muller C, Ward CR \& Wirtshafter DG 1984 Binding of mouse spermatozoa to the zonae pellucidae of mouse eggs in cumulus: evidence that the acrosomes remain substantially intact. Biology of Reproduction 31 1119-1128. (doi:10.1095/biolreprod31.5.1119)

Tulsiani DRP, Yoshida-Komiya H \& Araki Y 1997 Mammalian fertilization: a carbohydrate mediated event. Biology of Reproduction 57 487-494. (doi:10.1095/biolreprod57.3.487)

Visconti PE \& Florman HM 2010 Mechanisms of sperm-egg interactions: between sugars and broken bonds. Science Signaling 3 pe35. (doi:10. 1126/scisignal.3142pe35)

Yanagimachi R 2011 Mammalian sperm acrosome reaction: where does it begin before fertilization? Biology of Reproduction 85 4-5. (doi:10.1095/ biolreprod.111.092601)

Received 15 April 2011

First decision 20 May 2011

Accepted 5 July 2011 